\title{
Birational Finite Extensions of Mappings from a Smooth Variety
}

by

\author{
Marek KARAŚ \\ Presented by Wiesław PLEŚNIAK
}

Summary. We present an example of finite mappings of algebraic varieties $f: V \rightarrow W$, where $V \subset \mathbf{k}^{n}, W \subset \mathbf{k}^{n+1}$, and $F: \mathbf{k}^{n} \rightarrow \mathbf{k}^{n+1}$ such that $\left.F\right|_{V}=f$ and $\operatorname{gdeg} F=1<$ $\operatorname{gdeg} f$ (gdeg $h$ means the number of points in the generic fiber of $h$ ). Thus, in some sense, the result of this note improves our result in J. Pure Appl. Algebra 148 (2000) where it was shown that this phenomenon can occur when $V \subset \mathbf{k}^{n}, W \subset \mathbf{k}^{m}$ with $m \geq n+2$. In the case $V, W \subset \mathbf{k}^{n}$ a similar example does not exist.

1. Introduction. Let $\mathbf{k}$ be an algebraically closed field of characteristic zero, and let $f: V \rightarrow W$ be a finite mapping of algebraic subsets of $\mathbf{k}^{n}$ and $\mathbf{k}^{m}$, respectively. If $n \leq m$, then there exists a finite polynomial mapping $F: \mathbf{k}^{n} \rightarrow \mathbf{k}^{m}$ such that $\left.F\right|_{V}=f$ [10]. Let gdeg $h$ be the number of points in the generic fiber of a finite mapping $h$. A natural question is: what is the relation between gdeg $f$ and $\min \left\{\operatorname{gdeg} F \mid F: \mathbf{k}^{n} \rightarrow \mathbf{k}^{m}\right.$ finite such that $\left.\left.F\right|_{V}=f\right\}$ ? The answer to this question in several different situations is given in $[3]-[9]$.

If $n=m$, then $\operatorname{gdeg} F \geq \operatorname{gdeg} f$ for all finite extensions $F$ of $f$ [5]. The reason is that the image of $F$ is a normal variety (precisely, because $F: \mathbf{k}^{n} \rightarrow \mathbf{k}^{n}$ is finite, we have $\left.F\left(\mathbf{k}^{n}\right)=\mathbf{k}^{n}\right)$. But if $n<m$, then there is no obvious obstruction to existence of finite mappings $f: V \rightarrow W$ and $F: \mathbf{k}^{n} \rightarrow \mathbf{k}^{m}$ such that $\left.F\right|_{V}=f$ and $\operatorname{gdeg} F<\operatorname{gdeg} f$. For $m \geq n+2$ an example is given in [5]. It is natural to ask whether the similar phenomenon can occur when $m=n+1$. In this short note we give an affirmative answer to this question by proving the following 
THEOREM 1.1. Let $V \subset \mathbf{k}^{n}$ and $W \subset \mathbf{k}^{n+1}$ be smooth algebraic sets of dimension $k$, and let $f: V \rightarrow W$ be a finite mapping (possibly with gdeg $f$ large). If $2 k+1<n$, then there exists a finite mapping $F: \mathbf{k}^{n} \rightarrow \mathbf{k}^{n+1}$ such that $\left.F\right|_{V}=f$ and gdeg $F=1$ (that is, birational onto its image).

One can compare this theorem with the closely related results of M. Artin [1, Theorem (6.1)] and Srinivas [12], which however give the much higher dimension of the ambient space of $W$.

2. The proof. First of all recall that for any irreducible algebraic set $Z$, $k[Z]$ and $k(Z)$ mean, respectively, the ring of regular functions on $Z$ and the field of rational functions on $Z$. Recall also that a mapping $f: V \rightarrow W$ is called finite if $k[V]$ is integral over $f^{*}(k[W])$, where $f^{*}: k[W] \ni h \mapsto h \circ f \in$ $k[V]$, and that a polynomial mapping $f: V \rightarrow \mathbb{C}^{n}$ is called an embedding if $f(V)=\overline{f(V)}$ and $f$ is an isomorphism onto its image. We will need the following well known

LEMmA 2.1 (e.g. [2]). If $X \subset \mathbb{C}^{n}$ is a closed algebraic smooth set, $\operatorname{dim} X=k$ and $n>2 k+1$, then we can change the coordinates in such a way that the projection

$$
\phi: X \ni(x, y) \mapsto(0, y) \in 0 \times \mathbb{C}^{2 k+1}
$$

is an embedding.

By Lemma 2.1 we can assume that the projections

$$
\varphi_{1}: V \ni\left(x_{1}, \ldots, x_{n}\right) \mapsto\left(x_{1}, \ldots, x_{n-1}, 0\right) \in \mathbf{k}^{n-1} \times 0 \subset \mathbf{k}^{n}
$$

and

$$
\varphi_{2}: W \ni\left(y_{1}, \ldots, y_{n+1}\right) \mapsto\left(y_{1}, \ldots, y_{n-1}, 0,0\right) \in \mathbf{k}^{n-1} \times 0 \subset \mathbf{k}^{n+1}
$$

are embeddings. In this situation it is very easy and elementary to write down extensions $\Phi_{1} \in \operatorname{Aut}\left(\mathbf{k}^{n}\right), \Phi_{2} \in \operatorname{Aut}\left(\mathbf{k}^{n+1}\right)$ of $\varphi_{1}$ and $\varphi_{2}$, respectively. Indeed, for $\left(x_{1}, \ldots, x_{n-1}\right) \in \varphi_{1}(V)$, we have $\varphi_{1}^{-1}\left(x_{1}, \ldots, x_{n-1}\right)=$ $\left(x_{1}, \ldots, x_{n-1}, P\left(x_{1}, \ldots, x_{n-1}\right)\right)$ for some polynomial $P$. Thus

$$
\Phi_{1}: \mathbf{k}^{n} \ni\left(x_{1}, \ldots, x_{n}\right) \mapsto\left(x_{1}, \ldots, x_{n-1}, x_{n}-P\left(x_{1}, \ldots, x_{n-1}\right)\right) \in \mathbf{k}^{n}
$$

is the desired extension of $\varphi_{1}$. Similarly we construct $\Phi_{2}$.

Consider the mapping $\widetilde{f}=\varphi_{2} \circ f \circ \varphi_{1}^{-1}: \widetilde{V} \rightarrow \widetilde{W}$, where $\widetilde{V}=\varphi_{1}(V)$ and $\widetilde{W}=\varphi_{2}(W)$. Since $\widetilde{V} \subset \mathbf{k}^{n-1} \times 0 \subset \mathbf{k}^{n}$ and $\widetilde{W} \subset \mathbf{k}^{n-1} \times 0 \subset \mathbf{k}^{n+1}$, there exists a finite mapping $\widetilde{F}=\left(\widetilde{F}_{1}, \ldots, \widetilde{F}_{n-1}, 0,0\right): \mathbf{k}^{n-1} \times 0 \rightarrow \mathbf{k}^{n-1} \times 0$ such that $\left.\widetilde{F}\right|_{\widetilde{V}}=\widetilde{f}[10]$.

Let $h=a_{1} x_{1}+\cdots+a_{n-1} x_{n-1}$, where $a_{1}, \ldots, a_{n-1} \in \mathbf{k}$, be a primitive element of $\mathbf{k}\left(\mathbf{k}^{n-1}\right)=\mathbf{k}\left(x_{1}, \ldots, x_{n-1}\right)$ over $(\widetilde{F})^{*}\left(\mathbf{k}\left(\mathbf{k}^{n-1}\right)\right)=\mathbf{k}\left(\widetilde{F}_{1}, \ldots, \widetilde{F}_{n-1}\right)$ (see e.g. [11, Theorem A.7.1]). 
Put $\widehat{F}=\left(\widetilde{F}_{1}, \ldots, \widetilde{F}_{n-1}, x_{n}, x_{n} h\right): \mathbf{k}^{n} \rightarrow \mathbf{k}^{n+1}$. Then $\widehat{F}$ is a finite mapping. Indeed, since $\mathbf{k}\left[x_{1}, \ldots, x_{n-1}\right]$ is integral over $(\widetilde{F})^{*}\left(\mathbf{k}\left[\mathbf{k}^{n-1}\right]\right)=$ $\mathbf{k}\left[\widetilde{F}_{1}, \ldots, \widetilde{F}_{n-1}\right]$ (because $\widetilde{F}: \mathbf{k}^{n-1} \times 0 \rightarrow \mathbf{k}^{n-1} \times 0$ is finite), $x_{1}, \ldots, x_{n-1}$ are integral over $\mathbf{k}\left[\widetilde{F}_{1}, \ldots, \widetilde{F}_{n-1}\right]$, and so over $\mathbf{k}\left[\widetilde{F}_{1}, \ldots, \widetilde{F}_{n-1}, x_{n}, x_{n} h\right]$. Obviously $x_{n}$ is integral over $\mathbf{k}\left[\widetilde{F}_{1}, \ldots, \widetilde{F}_{n-1}, x_{n}, x_{n} h\right]$ too. This means that $\mathbf{k}\left[x_{1}, \ldots, x_{n}\right]$ is integral over $\mathbf{k}\left[\widetilde{F}_{1}, \ldots, \widetilde{F}_{n-1}, x_{n}, x_{n} h\right]$, i.e. $\widehat{F}$ is finite.

Also, $\widehat{F}: \mathbf{k}^{n} \rightarrow \widehat{F}\left(\mathbf{k}^{n}\right)$ is a birational mapping, because

$$
\mathbf{k}\left(x_{1}, \ldots, x_{n}\right)=\mathbf{k}\left(\widetilde{F}_{1}, \ldots, \widetilde{F}_{n-1}, x_{n}, x_{n} h\right)=(\widehat{F})^{*}\left(\mathbf{k}\left(\mathbf{k}^{n+1}\right)\right) .
$$

Indeed, we have

$$
h=\frac{x_{n} h}{x_{n}} \in \mathbf{k}\left(\widetilde{F}_{1}, \ldots, \widetilde{F}_{n-1}, x_{n}, x_{n} h\right) .
$$

Thus $\mathbf{k}\left(x_{1}, \ldots, x_{n-1}\right)=\mathbf{k}\left(\widetilde{F}_{1}, \ldots, \widetilde{F}_{n-1}, h\right) \subset \mathbf{k}\left(\widetilde{F}_{1}, \ldots, \widetilde{F}_{n-1}, x_{n}, x_{n} h\right)$. Since also $x_{n} \in \mathbf{k}\left(\widetilde{F}_{1}, \ldots, \widetilde{F}_{n-1}, x_{n}, x_{n} h\right)$, it follows that $\mathbf{k}\left(x_{1}, \ldots, x_{n}\right) \subset$ $\mathbf{k}\left(\widetilde{F}_{1}, \ldots, \widetilde{F}_{n-1}, x_{n}, x_{n} h\right)$, and so

$$
\mathbf{k}\left(x_{1}, \ldots, x_{n}\right)=\mathbf{k}\left(\widetilde{F}_{1}, \ldots, \widetilde{F}_{n-1}, x_{n}, x_{n} h\right) .
$$

Finally, because $x_{n} \in I(\widetilde{V})$, where $I(\widetilde{V})$ is the ideal of polynomials vanishing on $\widetilde{V}$, we have $\widehat{F}(x)=\left(\widetilde{F}_{1}(x), \ldots, \widetilde{F}_{n-1}(x), 0,0\right)=\widetilde{f}(x)$ for $x \in \widetilde{V}$. Thus $\widehat{F}$ is a birational finite extension of $\widetilde{f}$. Now it is easy to see that $\Phi_{2}^{-1} \circ \widehat{F} \circ \Phi_{1}$ is a birational finite extension of $f$.

\section{References}

[1] M. Artin, Algebraization of formal moduli: II. Existence of modifications, Ann. of Math. (2) 91 (1970), 88-135.

[2] Z. Jelonek, The extension of regular and rational embeddings, Math. Ann. 277 (1987), 113-120.

[3] M. Karaś, An estimate of the geometric degree of an extensions of some polynomial proper mappings, Univ. Iagel. Acta Math. 35 (1997), 131-135.

[4] —, Geometric degree of finite extension of projections, ibid. 37 (1999), 109-119.

[5] —, Birational finite extensions, J. Pure Appl. Algebra 148 (2000), 251-253.

[6] -, Finite extensions of mappings from a smooth variety, Ann. Polon. Math. 75 (2000), 79-86.

[7] - Finite extensions of mappings of finite sets, Bull. Polish Acad. Sci. Math. 50 (2002), 237-239.

[8] - Geometric degree of finite extensions of mappings from a smooth variety, J. Pure Appl. Algebra 212 (2008), 1145-1148.

[9] - , A note on geometric degree of finite extensions of mappings from a smooth variety, Bull. Polish Acad. Sci. Math. 56 (2008), 105-108.

[10] M. Kwieciński, Extending finite mappings to affine space, J. Pure Appl. Algebra 76 (1991), 151-153.

[11] S. Łojasiewicz, Introduction to Complex Analytic Geometry, PWN, Warszawa, 1988. 
[12] V. Srinivas, On the embedding dimension of the affine variety, Math. Ann. 289 (1991), 125-132.

Marek Karaś

Instytut Matematyki

Uniwersytet Jagielloński

Reymonta 4

30-059 Kraków, Poland

E-mail: Marek.Karas@im.uj.edu.pl

Received January 5, 2009;

received in final form June 4, 2009 